\section{NATIVOS DIGITALES EN COMUNIDADES VIRTUALES: UN ANÁLISIS DE LA INTERACCIÓN Y SOCIABILIDAD DE LOS ADOLESCENTES EN LA WEB EN EL CASO ARGENTINO}

\author{
DIGITAL NATIVES IN VIRTUAL COMMUNITIES: AN ANALYSIS OF \\ INTERACTION AND SOCIABILITY OF ARGENTINIAN ADOLESCENTS ON \\ SOCIAL NETWORKING WEBSITES ${ }^{1}$
}

\section{Marina Ollari ${ }^{2}$, Daniela Szpilbarg ${ }^{3}$, Juan Pablo Temelini ${ }^{4}$}

"No es Internet lo que cambia el comportamiento, sino que es el comportamiento el que cambia Internet".

Manuel Castells

\begin{abstract}
Institución y grupo del cual se desprende este trabajo: Área de Estudios Culturales: Instituto de Investigaciones Gino Germani (UBA)
\end{abstract}

Esta labor es un avance de un trabajo de investigación que se desarrolla en el marco del Área de Estudios Culturales del Instituto de Investigaciones Gino Germani, de la Universidad de Buenos Aires, dirigido por Ana Wortman ( http://www.iigg.fsoc.uba.ar/sitiosdegrupos/anawortman/) Este equipo lleva adelante un proyecto UBACYT 2008 /2010 titulado: Procesos de Globalización, Transformaciones Sociales y Redefinición del Campo Cultural en la Argentina contemporánea.

Palabras clave: web 2.0 - adolescentes- comunidades virtuales redes sociales- sociabilidad

Keywords: Web 2.0, adolescents, virtual communities, social networks, sociability.
Páginas 121 - 134
1 Traducción de Carlos A. Muñoz T. Revisión Centro de Traducción del Instituto de Idiomas UAM.

2 Licenciada en Sociología, Facultad de Ciencias Sociales, UBA. Auxiliar del equipo UBACYT Procesos de Globalización, Transformaciones Sociales y Redefinición del Campo Cultural en la Argentina contemporánea. m_ollari@yahoo.com.ar

3 Licenciada en Sociología, Facultad de Ciencias Sociales, UBA. Becaria doctoral Conicet. Participante del equipo de investigación del proyecto UBACYT Procesos de Globalización, Transformaciones Sociales y Redefinición del Campo Cultural en la Argentina contemporánea. danielaszpilbarg@hotmail.com

4 Licenciado en Sociología, Facultad de Ciencias Sociales, UBA. Auxiliar de investigación del equipo Ubacyt: Procesos de Globalización, Transformaciones Sociales y Redefinición del Campo Cultural en la Argentina contemporánea. jtemelini@ yahoo.com.ar

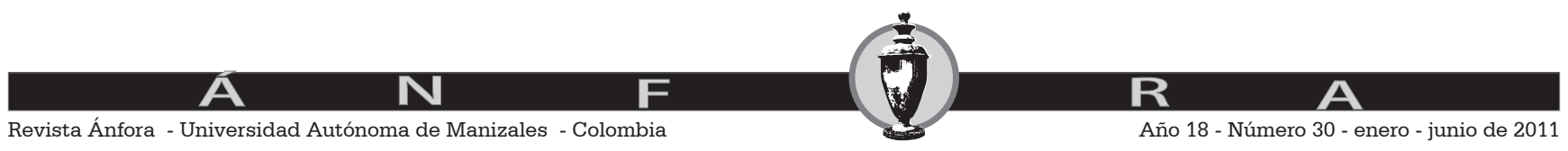




\title{
Resumen
}

Este trabajo intenta reflexionar acerca de Internet como medio de expresión y de creación comunitaria entre los adolescentes. Nuestro análisis se basará en cómo la web 2.0 propone nuevas formas de interacción en donde internet se constituye en un punto de encuentro. Entre los jóvenes, el uso comunicativo de internet es el más difundido. El MSN, los blogs y las redes sociales emergen como las formas predilectas de interacción social. Ante el debilitamiento de las instituciones tradicionales -como la familia y la escuela- el grupo de pares y las nuevas tecnologías avanzan como medios de sociabilidad. Las comunidades virtuales juegan un rol cada vez mayor en la construcción de la subjetividad de los jóvenes actuales, generación que entendemos como "nativos digitales". De esta manera, intentaremos explorar el modo en que los adolescentes se apropian de las tecnologías y reflexionaremos acerca de la sociabilidad que se genera a través de las comunidades virtuales en las que interactúan.

\begin{abstract}
This paper attempts to show some reflections about Internet as a means of expression and community creation among adolescents. Our analysis will be based on how the web 2.0 proposes new forms of interaction and becomes a meeting point. Among adolescents, the most spread use of Internet is the communicative one. MSN messenger, blogs and social networks emerge as the most favorite forms of social interaction. Due to the weakening of traditional institutions such as family and school, the group of pairs and new technologies overcome as a means of sociability. Virtual communities play an increasingly greater role in the construction of the subjectivity of modern youth, the so-called "digital natives" generation. Thus, we will explore the way in which adolescents appropriate technologies and will reflect about the sociability generated or yielded through virtual communities in which adolescents interact with others.
\end{abstract}

$E_{n}$ la era del individualismo, la vida privada y la autonomía personal, surge la necesidad de crear espacios cálidos, de mancomunión. Como bien lo expresó Bauman (2003: 6), la comunidad representa el tipo de mundo que todos queremos habitar, siendo que promete seguridad y mismidad. Claro que, es una idea cuyos valores se encuentran en constante tensión con los de libertad e individualidad.

En la actualidad, la web aparece como un espacio propicio para aunar ambas dimensiones, al menos durante el lapso de tiempo que estemos online.

Como dice Guinalíu (2003) al procurar definir los principales rasgos de las Comunidades Virtuales (CV) citando a varios autores (Whittaker, Issacs y O'Day, 1997) que coincidieron en lo siguiente:

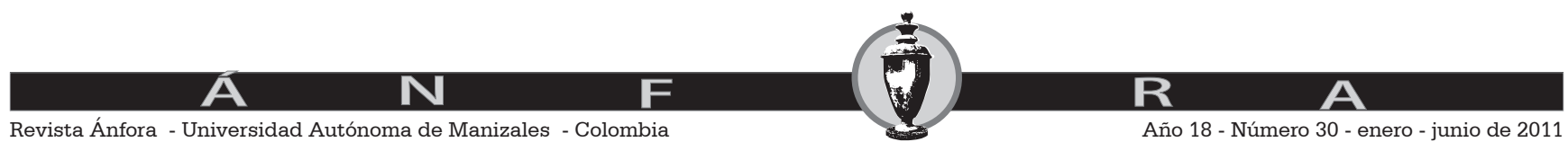


1. Los miembros comparten un objetivo, interés, necesidad o actividad que les ofrece la razón fundamental para pertenecer a la misma comunidad.

2. Los miembros tienen una actitud de participación muy activa, e incluso comparten lazos emocionales y actividades comunes muy intensas.

3. Los miembros poseen acceso a recursos compartidos, y políticas que rigen el acceso a esos recursos.

4. Existe recripocidad de información, soporte y servicios entre los miembros.

5. Los miembros comparten un contexto, un lenguaje y unas convenciones y protocolos (las denominadas "netiquette").

En este sentido, al hablar de adolescencia, no podemos dejar de hacer alusión al hecho de que internet resulta ser un medio ideal de expresión y creación comunitaria entre pares, en cuyo proceso terminan apropiándose de este espacio.

El término, Web 2.0 surge en 2004 para referirse a una segunda generación en la historia de la Web basada en comunidades de usuarios y una gama especial de servicios, como las redes sociales o los blogs, que fomentan la colaboración y el intercambio de información entre los usuarios. El uso de la Web 2.0 está orientado a la interacción y construcción de redes sociales, creando sitios interactivos. Es decir, que estos sitios actúan como puntos de encuentro a diferencia de los sitios web tradicionales. (Wikipedia, 2009).

De acuerdo a los resultados de la investigación "La Generación interactiva en Iberoamérica, Niños y adolescentes ante las pantallas" llevada a cabo por la Fundación Telefónica en 2008, el aspecto más importante para el cual se usa la conexión a Internet en todo Iberoamérica, son los "usos comunicativos" (redes sociales, programas de Chat, correo electrónico). En consecuencia, la relación social aparece como fin principal, y el medio más difundido es el uso del MSN, que encabeza el listado de servicios más utilizados entre los jóvenes. Se trata de una comunicación sincrónica, instantánea, llevada a cabo en tiempo real.

Veremos entonces, que las tecnologías serán una herramienta clave para desarrollar la conceptualización de un estilo y códigos propios, que hace las veces de una arena en dónde manifestarse lejos de la mirada de los adultos.

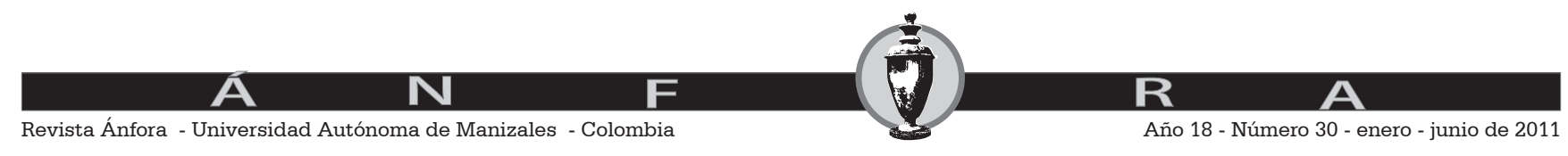




\section{Subjetividad adolescente y sociabilidad entre pares}

La personalidad del adolescente oscila entre actitudes que todavía son propias del niño que aún no ha dejado de ser y, al mismo tiempo, existe un anhelo de autonomía y emancipación que se relacionan con el adulto que quiere ser.

Más aún, el adolescente debe desarrollar su personalidad en medio de un contexto de desinstitucionalización e individualización que ocurre a nivel mundial, que se ve exacerbado en la Argentina post crisis 2001 ${ }^{5}$. Esta inestabilidad se observa en diferentes aspectos.

En primer lugar, la falta de un horizonte claro producto del devenir político y económico de la Argentina de los últimos años deposita a los jóvenes en un frágil y movedizo presente. Nada parece estar del todo claro porque nada permanece estable. Algunos factores como la precarización del mercado laboral, la falta de una política educativa duradera en el largo plazo y los vaivenes de la economía, posibilitan la emergencia de una subjetividad que debe afrontar el difuso devenir.

En segundo lugar, la desconfianza en depositar expectativas en las instituciones tradicionales, como la escuela y la familia, -que se debe a la falta de garantías que ellas ofrecen- configura lo imprevisible. Asimismo, la familia y la escuela han dejado de ser el principal núcleo de contención, y ambas se ven imposibilitadas en acompañar a los jóvenes en la socialización por medio de los nuevos canales que la modernidad ofrece. Al interior de la familia, la brecha generacional entre padres e hijos es cada vez mayor, debido a los constantes avances tecnológicos. En este sentido, los medios masivos de comunicación y los grupos de pares se han ido constituyendo como las instancias de socialización más influyentes en la vida cotidiana y, por lo tanto, en el proceso de subjetivación y formación de la identidad (Krauskopf, 2000).

En tercer lugar, el mercado asume un rol protagónico en cuanto a la distribución de recursos -tanto materiales como simbólicos- en detrimento del Estado que, lejos de constituirse como órgano central en la toma de decisiones, pierde prestigio social y moral como institución ante la cual poder confiar el futuro.

De esta manera, en la actualidad no es posible referirse a la subjetividad y sociabilidad adolescente sin explorar los usos y apropiaciones que éstos realizan de las nuevas tecnologías de información y comunicación (TIC).

Si bien a lo largo de este trabajo se hará mención a diversas TIC como la telefonía celular y la informática, nuestro análisis se centra en el uso de internet por parte de los jóvenes argentinos en los albores del Siglo XXI. Ser adolescente hoy supone, por un lado, la predisposición al uso de las tecnologías; y, por otro lado, la habilidad de poder explorarlas como verdaderos expertos.
5 El estallido económico y social producido a finales del año 2001 , nos enfrentó a varias consecuencias. Una de ellas fue la crisis institucional que afectó al Estado y a la clase política. El malestar social generalizado -por la profundización de la recesión económica, el aumento del desempleo y la pobreza y la implementación del "corralito" financiero- derivó en numerosas protestas que exigieron la renuncia del gobierno constitucional (Svampa, 2005).

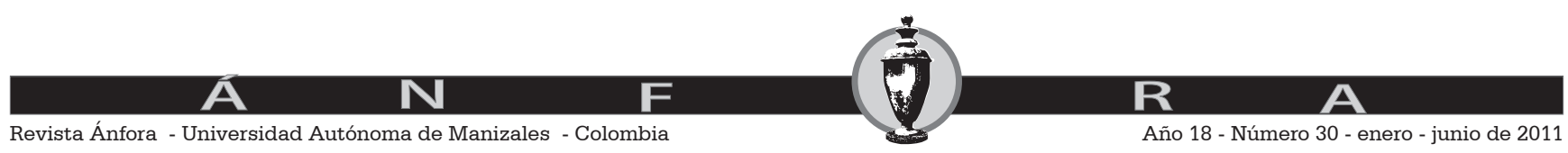


Como ya se ha dicho, los grupos de pares juegan un papel decisivo en la conformación de la identidad adolescente, en tanto funcionan como contención y aceptación en un período de angustia e inestabilidad emocional. Muchos autores han explorado la proliferación de diversas ciberculturas juveniles (Urresti, 2008), un ejemplo bien particular de ellas son los floggers (Ollari, Szpilbarg y Temelini, 2009), grupo conocido por su vinculación a las nuevas tecnologías que se destaca por su estilo de vida consumista.

Hoy en día, la mayoría de los adolescentes se encuentran en constante interacción con las tecnologías y la mayor parte de ellos tiene celular y correo electrónico. De esta manera, se produce una creciente interiorización de los implementos tecnológicos y una progresiva apropiación de las herramientas informáticas que posibilita la transformación de la subjetividad. Los jóvenes adquieren nuevas percepciones del tiempo y del espacio, y formas de aprender y de relacionarse entre ellos.

\section{Nativos digitales y redes sociales}

Resulta imprescindible analizar el concepto de "red social", ya que se presenta como componente ineludible de la interacción entre jóvenes cibernautas.

Tengamos en cuenta que las redes sociales pueden considerarse en sí mismas como formas de interacción social, y se definen como un intercambio dinámico entre personas, grupos e instituciones en contextos de complejidad. Un sistema abierto y en construcción permanente que involucra a conjuntos que se identifican en las mismas necesidades y problemáticas y que se potencian para organizar sus recursos. (Ministerio de Educación, 2009).

En este rasgo, los adolescentes se diferencian radicalmente de sus padres: tengamos en cuenta que sólo un $6 \%$ de los adultos son creadores de contenido, mientras que este porcentaje es del $40 \%$ en el caso de adolescentes (Fundación Telefónica, 2008).

Marc Prensky (2001: 1) plantea la diferencia en los usos de las TIC en términos de brecha generacional, al introducir el término nativos digitales para definir a quienes nacieron en un mundo constituido por y alrededor de tecnologías digitales, una tecnología diferente y distante de las que enmarcaron la vida de los adultos de la generación anterior. Para Prensky, esta circunstancia, ha generado una brecha entre una y otra generación, los "nativos" (que nacieron en su entorno) y los "inmigrantes", adultos para quienes a esta tecnología les adviene en sus vidas. Este autor plantea que "Nuestros estudiantes han cambiado radicalmente... Los estudiantes de hoy representan la primera generación que creció con esta nueva tecnología (digital). Han pasado sus vidas enteras usando y rodeados por las computadoras, los juegos de video, los reproductores de música digitales, las videocámaras, los teléfonos celulares, y el resto de aparatos y herramientas de la era digital. (...) han pasado menos

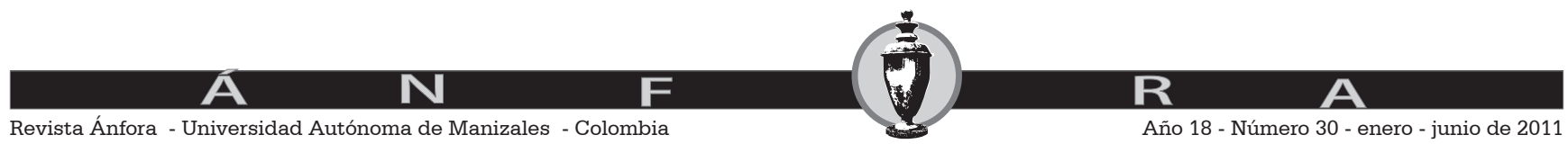


de 5.000 horas de sus vidas leyendo, pero durante 10.000 horas divertidos en los juegos video (sin mencionar 20.000 horas que han visto TV). Los juegos de la computadora, el email, Internet, los teléfonos celulares y la mensajería instantánea MSN son partes de sus vidas.

Para Alejandro Piscitelli (2006), los "nativos digitales", estarían emergiendo como una nueva clase cognitiva, algo que va bastante más allá del uso "naturalizado" de las tecnologías digitales de época. La inmersión en la tecnocultura, las prácticas sociales derivadas en tanto ambiente, estarían generando una brecha cognitiva generacional.

Vale entonces preguntarnos en qué consisten estas prácticas que desembocan en una especificidad cognitiva propia de los nativos digitales.

Y retomando la idea planteada en el comienzo del artículo respecto a que Internet resulta un espacio de intersección entre lo comunitario y lo individual, es interesante hacer alusión a una reflexión que hace Manuel Castells (1999) -al citar un estudio de Barry Wellman- en donde destaca que las comunidades virtuales en Internet son comunidades en tanto generan sociabilidad, relaciones y redes de relaciones humanas. Sin embargo, estas comunidades se diferencian de las comunidades físicas y poseen su propia lógica. Más aún, la tendencia actual es hacia la disminución de la sociabilidad de base comunitaria física tradicional.

De modo que la pregunta planteada parece ser ¿Qué tipo de relaciones y prácticas genera?

\section{Usos y valoraciones}

En el informe de la Asociación Civil Chicos.net (2008) surgen algunos datos porcentuales acerca de los usos de las herramientas informáticas que hacen chicos de entre 9 y 18 años. Este estudio, además, desagrega estos porcentajes analizándolos en relación a la edad y el nivel socioeconómico de los chicos encuEstados.

Si tenemos en cuenta simplemente el uso de Internet, el 40,2\% de los chicos y jóvenes encuEstados dijo utilizar Internet todos los días de la semana. Los que se conectan a Internet todos los días de la semana son mayoritariamente los adolescentes (15 a 18 años), en donde el 60\% de ellos lo hace todos los días, mientras que de los chicos de 9 a 11 años se conecta con esa frecuencia el $27,9 \%$.

A medida que aumenta la edad, los jóvenes van interiorizando las herramientas tecnológicas: se observa que de los chicos de 9 a 11 años un $71,3 \%$ posee mail, mientras que en el grupo de 12 a 14 años esto ocurre en el $84 \%$ de los casos; y en los chicos de 15 a 18 años el 93,4\% tiene mail.

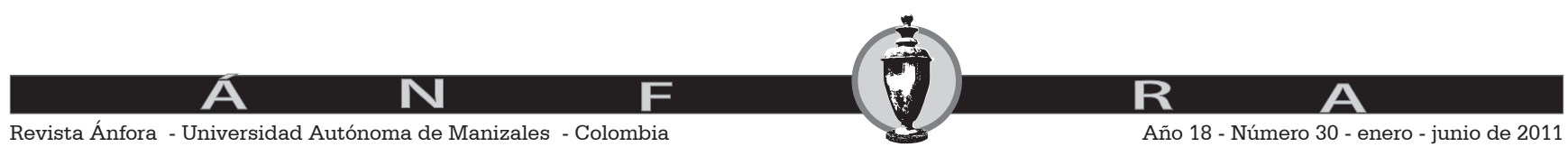


Respecto de las herramientas interactivas y de creación de contenidos, como el fotolog y el blog, de los chicos encuEstados, el 37\% tiene fotolog y/o blog. Al comparar la posesión de fotolog/blog según nivel socioeconómico, podemos ver que casi la mitad de los chicos de nivel medio alto tiene $46,7 \%$ mientras que en el caso del nivel medio bajo, menos de la cuarta parte lo tiene $24,4 \%$, lo cual nos permite pensar en la vinculación de creación de contenidos con el tener acceso a Internet y a ciertos consumos.

Comparando los sexos, la diferencia es notable: el 44,6\% de las mujeres posee fotolog o blog, mientras que sólo el $29 \%$ de los varones lo hace.

\section{Sociabilidad y comunicación}

Hay que pensar de qué modo se configura la identidad y la sociabilidad de los chicos que poseen fotolog y blog y en qué medida ésto afecta a sus relaciones sociales, teniendo en cuenta que es probable que, al circular en la virtualidad gran parte del tiempo y que gran parte de los intercambios sociales se den en este ámbito, exista una exigencia implícita de ser parte de esta red.

Según la edad, podemos ver que el $24,8 \%$ de los chicos de 9 a 11 años tiene fotolog/blog, mientras que conforme aumentan en edad, más chicos lo tienen: en el caso de los 12 y 14 años, el 39\% posee fotolog y entre los 15 a 18 años, la cantidad asciende al 52,8\%. (Chicos Net, 2006).

El 24,7\% de los chicos pertenece a una comunidad virtual como Facebook o Myspace. En cuanto a las edades de los chicos que pertenecen a alguna de estas "comunidades" virtuales, sólo el 11,7\% de 9 a 11 años lo hace, mientras que entre los 15 y 18 el 40,4\% pertenece a una de estas comunidades virtuales. Respecto a la diferencia por nivel socioeconómico, ésta es marcada: el 37,5\% de los de nivel socioeconómico alto pertenece a una comunidad virtual, mientras que sólo el 17\% de los chicos de nivel medio y el $12,9 \%$ de los de nivel medio bajo y bajo lo hace. Aquí también es evidente que la pertenencia a la comunidad virtual requiere un uso cotidiano de las herramientas informáticas.

Los chicos de NSE medio alto poseen computadora con conexión a Internet en el hogar en un $96,6 \%$ de los casos, mientras que ésto sólo ocurre en el 34\% de los hogares de de NSE medio bajo y bajo, y en el 63,3\% de los de nivel medio medio. Los datos sobre la conexión por banda ancha reflejan también esta situación: un 72,8\% de los chicos y adolescentes de NSE medio alto tienen conexión por banda ancha, mientras que esto ocurre en el 37,8\% de los casos en NSE medio medio y en el $12,3 \%$ de NSE medio bajo y bajo.

Varias encuestas realizadas en los últimos tiempos revelan datos semejantes a la hora de hablar de la interacción cada vez más constante de los adolescentes en el entorno web: la consultora D'Alessio- Irol (2006) realizó un estudio en el cual analiza la relación de los jóvenes con Internet: los resultados arrojan que siete de cada diez "no puede vivir sin internet". Este estudio indagó

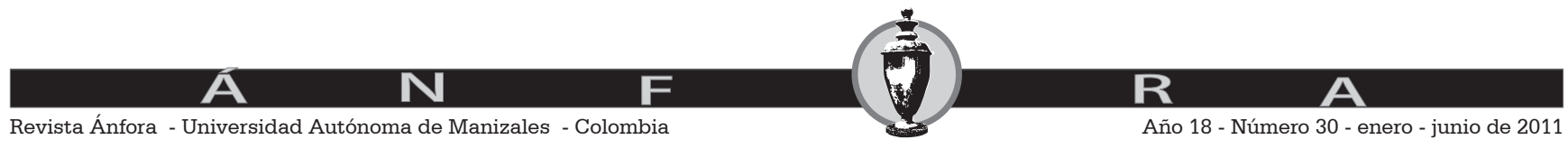


en las opiniones de mil jóvenes de seis países latinoamericanos y comprobó que aparecen como "super tecnificados" poseyendo el $80 \%$ de ellos celular.

Se destaca que las razones que esgrimen los jóvenes son que ahorra tiempo, es útil y facilita la comunicación. (Fundación Telefónica, 2008). Según los encuEstados, la mensajería instantánea (MSN y Chat) permite una comunicación continua, sencilla y barata con sus pares. Más aún, el MSN permite un mayor control de la lista de contactos y bloquear el acceso a desconocidos.

Argentina se distingue por ser uno de los países con mayor predominancia del uso "para comunicar" (MSN y correo electrónico). Sin embargo, es también uno de los países donde los salones de Chat son menos populares. Esto significaría que los usuarios de MSN prefieren comunicarse con "conocidos". Cabe señalar que el mayor uso empieza a crecer a partir de los 12 años de edad, momento en el cual los chicos comienzan el paso a la adolescencia y aumenta su necesidad de sentirse integrados en un grupo de pares con los cuales se identifican.

Por lo general, se busca el contacto con un grupo preexistente, pero en las encuestas es notorio que Internet aparece como un espacio propicio para conocer a nuevas personas. En relación a esto, dos de cada tres usuarios tienen amigos que conocieron en entornos virtuales y uno de cada tres llegó a conocer a su colega virtual en persona.

\section{Control parental}

En cuanto a los adultos, por otro lado, la relación pasa por el control parental, que es variable. Por una parte hay un rol más activo, que implica control y acompañamiento simultáneo en el momento del uso de la web: navegar juntos, estar en la misma habitación, preguntarle lo que hace. Por otra parte, existe un control de tipo pasivo: posterior, de revisión de las páginas visitadas, de los historiales y actividades realizadas. Comprobar por dónde navegó, mirar el email.

Como porcentaje general, en un $36 \%$ de los casos, los padres no hacen nada. Argentina se destaca por tener una gran autonomía de los jóvenes con respecto a sus padres (porcentajes superiores a 57\% en los mayores a 14 años). Es muy poco frecuente la navegación conjunta de padres e hijos (Fundación Telefónica, 2008).

Por otra parte, la encuesta "Internet en los hogares" realizada entre 2007 y 2008 por la Sociedad Argentina de Pediatría, revela que en la mayoría de los casos los padres no se involucran en el control y supervisión que deberían llevar a cabo de las acciones de sus hijos en relación con las nuevas tecnologías: un 53\% de los padres refirió que conversa con sus hijos acerca de la Web, mientras que el $47 \%$ restante lo hace "a veces o nunca". Asimismo, es

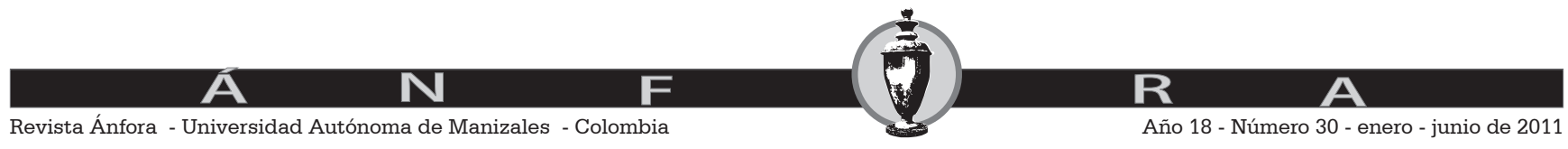


notable que en el $23 \%$ de los casos, en el hogar no hay regla alguna respecto al uso de Internet.

En relación con estos datos, del estudio realizado por Chicos.Net en 2008, podemos observar que sólo un 1,6\% de los encuEstados entre 9 y 18 años contestó que hay un adulto presente siempre cuando el chico navega por Internet, mientras que el $44 \%$ contestó que nunca hay un adulto presente acompañando. El 21,4\%, sin embargo, contestó que a veces hay adultos presentes cuando navegan por Internet. Entre quienes contestaron que a veces hay un adulto presente, el $26,5 \%$ son chicos de 9 a 11 años, mientras que esto ocurre en el 12\% de los chicos de 15 a 18 años. En este grupo, un 60,8\% dijo que nunca hay un adulto presente cuando navega.

Respecto a cómo aprendió a encontrar sitios web que le interesen, las dos opciones más elegidas fueron: aprendió solo $61,4 \%$ y le enseñó un amigo $22 \%$. La opción menos elegida es la de los padres como figuras que le ayudaron al chico en el uso de Internet. Es notable por un lado la importancia para los chicos, de sus pares, y por otro lado, la idea del "aprendió solo" se relaciona con la interiorización que los chicos tienen de las tecnologías. Quienes más eligieron a los padres como las figuras que los ayudaron a encontrar sitios web de su interés, son los chicos de entre 9 y 11 años. También hay diferencias según el NSE: un 15,4\% de los chicos de NSE medio alto eligió esta opción mientras que sólo lo hizo un $5 \%$ de chicos y jóvenes de NSE medio bajo y bajo. Puede pensarse que los padres de los chicos de estratos más altos están más "pendientes" de los comportamientos de sus hijos.

Ante un conflicto respecto del uso de Internet, la causa más fuerte suele ser la cantidad del tiempo, pero no lo que se hace en la web. Asimismo, también entra en discusión el momento del día en que se conectan. No se discuten el uso, los contenidos y las acciones.

\section{Ocio tradicional vs ocio interactivo}

Lo que muestran las encuestas es que a mayor edad, mayor preferencia por el interactivo. (Fundación Telefónica, 2008). Más aún, es curioso el hecho de que los jóvenes afirman tener más afinidad por Internet, siendo que la televisión es el medio masivo por excelencia.

En este sentido, se pone sobre el tapete la variable socioeconómica, es decir, que no todos los jóvenes tienen la posibilidad de acceder a Internet. Sin duda esta posibilidad de elegir entre dos tipos de pantallas (alternativas de ocio) sólo existe en un sector de la población, para otros sin embargo, la televisión aparece como la única pantalla disponible en el hogar.

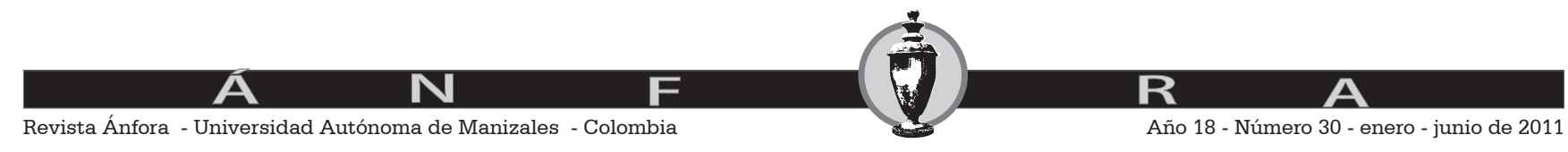




\section{Comunidades Virtuales: el caso flogger}

Para ejemplificar la temática en relación a la sociabilidad de los jóvenes mediada por las nuevas tecnologías y la conformación de comunidades virtuales, tomaremos el caso paradigmático de los floggers en Argentina. Esta comunidad de adolescentes es por antonomasia un grupo construido a partir del uso de las nuevas tecnologías. La denominación flogger proviene del hecho de poseer un fotolog: página web personal que permite publicar fotos y texto, y a la vez recibir los comentarios de sus pares en un formato estandarizado ${ }^{6}$. El sitio fue abierto al público en el año 2002 y en la actualidad posee más de 20 millones de usuarios en todo el mundo, y se calcula que hacia fines de 2007 eran 2.084.000 los usuarios argentinos de Fotolog.com (Ollari, Szpilbarg y Temelini, 2009). La comunidad flogger se caracteriza por el culto a la imagen y por mostrar una versión estética y juvenil de sí mismos.

Si tenemos en cuenta la definición de Comunidad Virtual a la que Guinaliú (2003) hace referencia citando a Preece (2000), bien podemos decir que los floggers son un claro ejemplo de comunión en la red a partir de gustos e intereses particulares: (1) gente que desea interactuar para satisfacer sus necesidades o llevar a cabo roles específicos, (2) que comparten un propósito determinado (un interés, una necesidad, un servicio o un intercambio de información) que constituye la razón de ser de la CV, (3) con una política que guía las relaciones y (4) con unos sistemas informáticos que median las interacciones y facilitan la cohesión entre los miembros.

En este sentido, los floggers emergen como un grupo de jóvenes que poseen ciertas características:

Por un lado, se destaca su vinculación a las nuevas tecnologías, "estar en la compu" es estar comunicado con el grupo de pares. La sociabilidad aparece mediada por internet y, al mismo tiempo, esto genera consecuencias en la dinámica que adquiere el mismo medio. La generalización del uso comunicativo de internet por parte de los jóvenes aumenta la oferta de sitios y redes sociales que ofrecen la posibilidad de establecer contactos. Claro que, en el marco de las reglas pautadas por fotolog.com.

Por otro lado, los floggers practican un estilo de vida consumista que se observa, por ejemplo, en el uso de espacios territoriales ubicados en el centro más que en la periferia de la vida social. Uno de los aspectos más llamativos es la elección de los shoppings como núcleo de encuentro. Asimismo, los jóvenes floggers se identifican a sí mismo como una moda, lo que conlleva una carga temporaria de fugacidad, además de reafirmar el aspecto consumista.

Finalmente, podríamos concluir que, en este caso particular, las pautas culturales que enmarcan a la comunidad flogger se corresponden con los valores que propone la sociedad de consumo. En una sociedad que produ-
6 El sitio Fotolog.com fue creado por Scott Heiferman y Adam Seifer y nació como una red de 200 personas que compartían fotos.

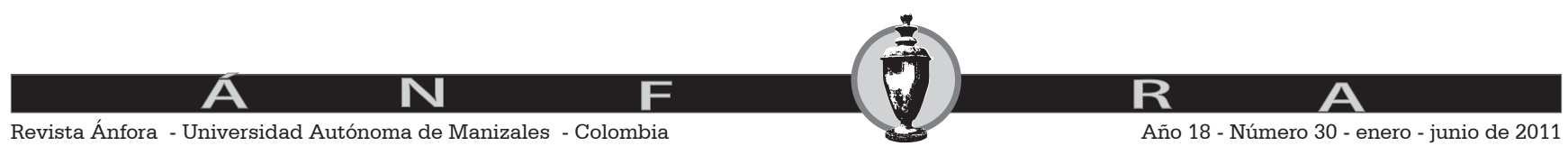


ce símbolos en serie el esfuerzo es por distinguirse: los jóvenes floggers exaltan el consumo de los bienes y símbolos que proponen las grandes marcas a través del marketing y la publicidad, acatando las normas éticas de la sociedad de consumo.

Lo interesante de este caso es que si bien surgió como una comunidad meramente virtual, supo extender esta interacción a "la vida real" a partir de encuentros en shoppings, en donde los vínculos generados mantuvieron las jerarquías e identificaciones creadas a partir de la web.

\section{Reflexiones finales}

A partir de los datos analizados, podemos hacer una síntesis de los usos e interacciones que se dan en el espacio de la web.

En principio vemos que a medida que los jóvenes llegan a la adolescencia, es mayor el uso de internet y, dentro de este marco, aumenta el uso de programas y aplicaciones que implican cierta creatividad. Se exacerba el uso de fotologs y blogs, que requieren una práctica "activa" e interactiva y por ende cierta apropiación de la herramienta.

Por otra parte, a medida que aumenta la edad, los jóvenes utilizan la web priorizando su función social y comunicativa. Así, crece la creación de cuentas en comunidades virtuales y la asociación a redes sociales como el facebook, mientras que el MSN y el Chat son los preferidos para la comunicación instantánea.

Algunas de las razones para estos comportamientos se deben a la facilidad de acceso que implica internet, así como la familiaridad en el uso de esta herramienta en tanto estos jóvenes forman parte de los denominados nativos digitales. Por otra parte, es destacable el factor generacional, que le da a estos jóvenes un empoderamiento en relación a los usos y conocimientos que tienen sus padres. Estos últimos, por otra parte, parecen tener un rol mayoritariamente pasivo de control de los usos que sus hijos hacen de internet, lo cual le da a los adolescentes gran autonomía en este medio.

Claro está que la relación de los jóvenes con internet se ve cruzada por la dimensión socioeconómica. En general, se mantiene este crecimiento en el uso de Internet teniendo en cuenta la variable edad (a mayor edad mayor uso), pero se ve una clara disminución al tener en cuenta la variable de clase social (menor uso a menor NSE).

Internet se presenta así, como un espacio de expresión e interacción entre pares, propicio para desarrollar su sociabilidad y la construcción identitaria.

“(...) son comunidades personales, comunidades de personas basadas en los intereses individuales y en las afinidades y valores de las personas. Es decir,

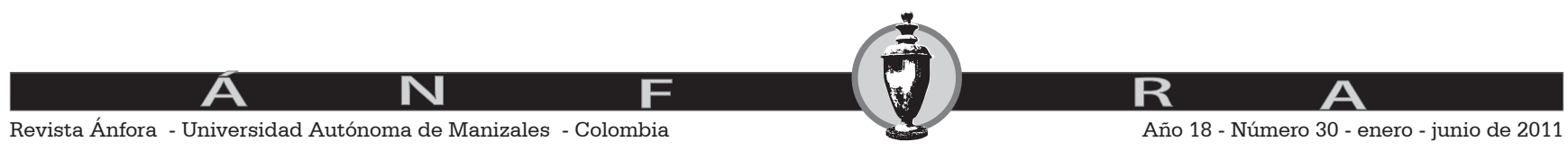


en la medida en que se desarrollan en nuestras sociedades proyectos individuales, proyectos de dar sentido a la vida a partir de lo que yo soy y quiero ser, Internet permite esa conexión saltando por encima de los límites físicos de lo cotidiano, tanto en el lugar de residencia como en el lugar de trabajo, y genera, por tanto, redes de afinidades". (Manuel Castells, 1999: 14).

Estas reflexiones nos abren las puertas para seguir explorando nuevas formas de sociabilidad entre los jóvenes. Quedan planteados nuevos interrogantes: ¿qué ocurre con la sociabilidad mediada por la tecnología en diferentes estratos sociales? O bien, ¿cuáles son las tecnologías que más intervienen en la sociabilidad de los jóvenes por nivel socioeconómico? Y, por otro lado, ¿en qué medida la pertenencia a un sector social determinado incide en los vínculos con las nuevas tecnologías?

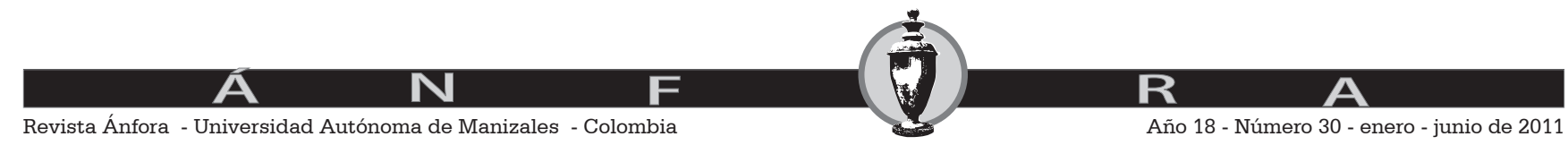




\title{
Bibliografía
}

\begin{abstract}
-ASOCIACIÓNCIVILCHICOS.NET.BALARDINI,S.YOTROS(2008):Chic@s y Tecnología Usosycostumbresdeniñas, niñosyadolescentesenrelaciónalas Tecnologías de Información y la Comunicación, Buenos Aires. Disponible en sitio Save the Children Suecia. http://www.scslat.org/web/ publicaciones/interna.php?xopcp $=5 \&$ xid $=321 \&$ xleng $=e$
\end{abstract}

- BAUMAN, Z. (2006): Comunidad: en busca de seguridad en un mundo hostil. Ed. Siglo XXI, Madrid.

- CASTELLS,M.(2000):"Internet y la sociedad red". En: Lección inaugural del programa de doctorado sobre la Sociedad de la Información y el Conocimiento [conferencia en línea]. UOC

- D'LESSIO IROL (2006): Internet en la Argentina. http://200.32.3.17/ archivos/Internet_en_argentina_2005_2006.zip

- FUNDACIÓN TELEFÓNICA (2008): La Generación Interactiva en Iberoamérica, Niños y adolescentes ante las pantallas. Ed. Ariel, Barcelona.

- GUINALIÚ, M. (2003): "La Comunidad Virtual", [en línea] 5campus. org, Sistemas Informativos Contables <http://www.ciberconta.unizar. es/leccion/comunidades $>$

- KRAUSKOPF, D. (2000): "Procesos psicológicos centrales en la adolescencia" en Participación social y desarrollo en la adolescencia. UNFPA, San José de Costa Rica.

-MINISTERIO DE EDUCACIÓN (2009): "Redes sociales: interacción social en Internet". En Boletín elctrónico de la Biblioteca del docente. Año 4 Número 19.

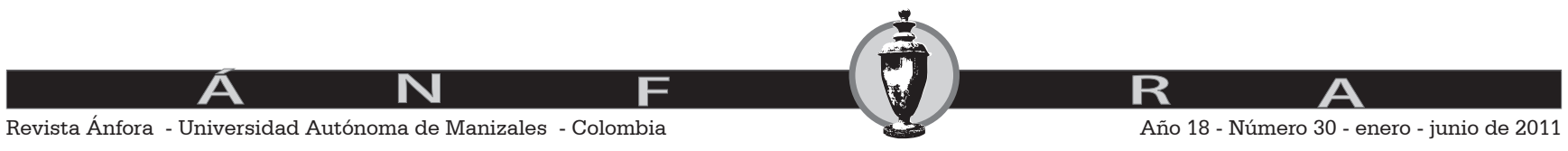


- OLLARI, M.; SZPILBARG, D.; TEMELINI, J. (2009): "La influencia de las TIC en la conformación de nuevas subjetividades y formas de sociabilidad entre los adolescentes: el caso de los floggers del Abasto", ponencia presentada en el XXVII Congreso Alas, Buenos Aires.

- PISCITELLI, A. (2006) "Nativos e inmigrantes digitales". En RMIE, VOL 11, Núm 28

http://www.scribd.com/doc/467656/Nativos-e-Inmigrantes-Digitales

-PRENSKY, M. (2001): "Digital Natives, Digital Immigrants". On the Horizon, NCB University Press, Vol. 9 No. 5.

- SOCIEDAD ARGENTINA DE PEDIATRÍA (2007/2008): Encuesta "Internet en los hogares", Buenos Aires.

- SVAMPA, M. (2005): La Sociedad Excluyente. La Argentina bajo el signo del neoliberalismo. Taurus, Buenos Aires.

- URRESTI, M. (ed.) (2008): Ciberculturas juveniles. Los jóvenes, sus prácticas y sus representaciones en la era de Internet. La Crujía, Buenos Aires.

-WIKIPEDIA. http://es.wikipedia.org/wiki/Web_2.0

- WINOCUR, R (2006): "Internet en la vida cotidiana de los jóvenes" en Revista Mexicana de Sociología 68, Nro. 3.

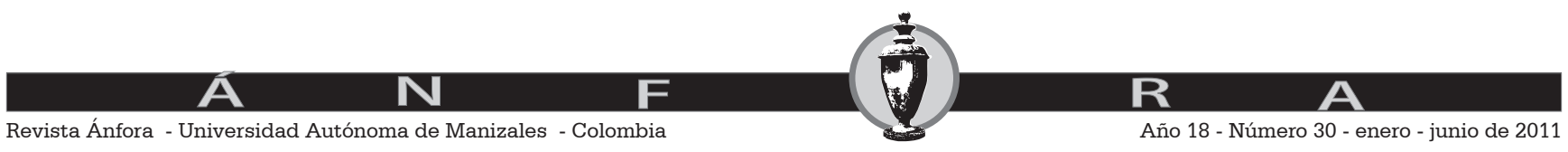

